\title{
Effects of rotation in the laser-pulse photoassociation in a thermal gas of atoms
}

\author{
Emanuel F. de Lima \\ Instituto de Geociências e Ciências Exatas, UNESP - Univ Estadual Paulista, Av. 24A 1515, \\ CEP 13506-900, Rio Claro, São Paulo, Brazil
}

\begin{abstract}
Photoassociation is a possible route for the formation of chemical bonds. In this process, the binding of colliding atoms can be induced by means of a laser field. Photoassociation has been studied in the ultracold regime and also with temperatures well above millikelvins in the thermal energy domain, which is a situation commonly encountered in the laboratory. A photoassociation mechanism can be envisioned based on the use of infrared pulses to drive a transition from free colliding atoms on the electronic ground state to form a molecule directly on that state. This work takes a step in this direction, investigating the laser-pulse-driven formation of heteronuclear diatomic molecules in a thermal gas of atoms including rotational effects. Based on the assumption of full system controllability, the maximum possible photoassociation yield is deduced. The photoassociation probability is calculated as a function of the laser parameters for different temperatures. Additionally, the photoassociation yield induced by subpicosecond pulses of a priori fixed shape is compared to the maximum possible yield.
\end{abstract}

Keywords: photoassociation, laser pulses, rotation, thermal gas

\section{INTRODUCTION}

Photoassociation reactions have been attracting considerable interest. ${ }^{1}$ In this process, two colliding atoms are bound through the absorption of a photon. The binding of the atoms is often achieved by means of a laser field, which induces the free-to-bound transition. Photoassociation can be viewed as a fundamental chemical reaction catalyzed by the laser field and controlling this process is a possible means for inducing new chemical reactions. ${ }^{2}$

In the cold and ultracold regimes, photoassociation has been used as a pathway to form cold molecules from previously cooled atoms. Usually, this process involves the absorption of visible or ultraviolet radiation by the colliding atoms to form a molecule in an electronic excited state. However, if the colliding atoms are from two different species such that there exist a non-negligible permanent dipole coupling between the free atomic states and the rovibrational levels of the molecule ground state, an alternative photoassociation mechanism can be envisioned based on infrared laser pulses. ${ }^{3}$ In this mechanism, the infrared laser can drive a transition from the free atomic state directly to the electronic bound state of the molecule to be formed.

Photoassociation reactions of hot atoms (temperatures well above millikelvins) have also been demonstrated experimentally. ${ }^{4,5}$ Investigating photoassociation in this regime is very important, since this is a situation more often encountered in the laboratory. In the present work, we investigate photoassociation in a thermal gas composed of two different atomic species. We extend a previous work ${ }^{3}$ by including the rotational degree of freedom, since in the thermal condition, many partial waves may participate in the photoassociation process. The photoassociation probability is obtained by incoherently averaging the transitions from Boltzmann-weighted scattering levels. Our goal is to study the effects of rotation in the photoassociation probability. Based on the assumption of full system controllability, the maximum possible photoassociation yield is deduced. Considering subpicosecond pulses with a priori fixed shape, the photoassociation probability is calculated for different temperatures and as a function of the laser field parameters, while these results are compared to the maximum possible yield.

Further author information: E-mail: eflima@rc.unesp.br, Telephone: 55-19-35269134

8th Iberoamerican Optics Meeting and 11th Latin American Meeting on Optics, Lasers, and Applications, edited by Manuel Filipe P. C. Martins Costa, Proc. of SPIE Vol. 8785, 878527

(C) 2013 SPIE · CCC code: $0277-786 X / 13 / \$ 18 \cdot$ doi: $10.1117 / 12.2024968$

Proc. of SPIE Vol. $8785878527-1$ 


\section{PHOTOASSOCIATION IN A THERMAL GAS OF ATOMS}

Description of the Model. Consider a dilute gas of atoms composed of two distinct atomic species at translational thermal equilibrium and subjected to a linearly-polarized infrared laser pulse. Assuming that only binary collisions take place, the goal is to determine the probability of forming heteronuclear diatomic molecules induced by the laser pulse. Before the action of the laser pulse, the density operator of two colliding atoms in the gas is

$$
\rho(0)=\frac{1}{Z} \mathrm{e}^{-\beta H_{0}}
$$

where $H_{0}$ is the field-free Hamiltonian, $Z$ is the partition function and $\beta=1 / k_{\mathrm{B}} T$ with $k_{\mathrm{B}}$ being the Boltzmann constant and $T$ the temperature. The field-free Hamiltonian for the relative motion of the nuclei is

$$
H_{0}=-\frac{\hbar^{2}}{2 M} \frac{1}{r} \frac{\partial^{2}}{\partial r^{2}} r+\frac{\mathbf{L}^{2}}{2 M r^{2}}+V(r)
$$

with $r=|\vec{r}|$ being the separation of the two nuclei, $M$ the associated reduced mass, L the angular momentum operator and $V(r)$ the interatomic potential. This potential is given by a Morse oscillator, $V_{\mathrm{M}}(r)=$ $D_{e}\left\{\exp \left[-2 \alpha\left(r-r_{e}\right)\right]-2 \exp \left[-\alpha\left(r-r_{e}\right)\right]\right\}$, for $r<L$ and is truncated at $r=L$ by a infinite barrier. ${ }^{6}$ The introduction of the barrier allows us to perform all calculations with square integrable eigenstates as basis functions. Upon increasing the position of the barrier, the discretized unbound levels approximate a continuum of states.

The interaction of the colliding atoms with a linearly-polarized homogeneous electrical field is given by

$$
H_{1}=-\mu(r) \varepsilon(t) \cos (\theta)
$$

where $\mu(r)$ is the permanent dipole associated with the collision process and $\theta$ is the angle between the electric field polarization vector and $\vec{r}$. The dipole function has the form $\mu(r)=q r \mathrm{e}^{-r / r_{d}}$, where $q$ is the dipole effective charge and $r_{d}$ specifies the range of the dipole interaction. The electric field of the laser is a pulse that starts at $t=0$ and ends at $t=t_{f}$ with a sine-squared envelope given by $\epsilon(t)=\epsilon_{0} \sin ^{2}\left(\pi / t_{f}\right)$ sin $\omega t$, where $\epsilon_{0}$ is the amplitude and $\omega$ the carrier frequency.

We only consider initial states with $m_{\ell}=0$, where $m_{\ell}$ is the number of the $2 \ell+1$ rotational sublevels. Since $m_{\ell}$ is conserved for the linear polarization of the incident laser radiation, the wavefunction of a pure state can be written as

$$
\langle r \mid \psi(t)\rangle=\psi(\vec{r}, t)=\sum_{\nu=1}^{\infty} \sum_{\ell=0}^{\infty} a_{\nu, \ell}(t) Y_{\ell}^{0}(\theta) \phi_{\nu \ell}(r) / r
$$

where $Y_{\ell}^{m_{\ell}}$ are the spherical harmonics and the radial wavefunctions $\phi_{\nu \ell}(r)$ are solutions of the eigenvalue equation

$$
\left[-\frac{\hbar^{2}}{2 M} \frac{d^{2}}{d r^{2}}+\frac{\hbar^{2} \ell(\ell+1)}{2 M r^{2}}+V(r)\right] \phi_{\nu \ell}(r)=E_{\nu \ell} \phi_{\nu \ell}(r)
$$

where $E_{\nu \ell}$ are the corresponding energies of the rotational-vibrational levels.

Substitution of Eq.(4) into time-dependent Schrödinger equation yields the following equations for the coefficients $a_{\nu \ell}(t)$,

$$
\mathrm{i} \hbar \frac{d a_{\nu \ell}}{d t}=E_{\nu, \ell} a_{\nu \ell}-\varepsilon(t) \sum_{\nu^{\prime}=1}^{\infty}\left[a_{\nu^{\prime} \ell+1}\left\langle\phi_{\nu^{\prime} \ell+1}|\mu| \phi_{\nu \ell}\right\rangle p_{\ell}+a_{\nu^{\prime} \ell-1}\left\langle\phi_{\nu^{\prime} \ell-1}|\mu| \phi_{\nu \ell}\right\rangle p_{\ell-1}\right]
$$

where $^{7}$

$$
p_{\ell}\left\langle Y_{\ell+1}^{0}|\cos (\theta)| Y_{\ell}^{0}\right\rangle=\frac{\ell+1}{\sqrt{(2 \ell+1)(2 \ell+3)}}
$$


For effective numerical calculations, the infinite summations in the expansion (4) are truncated to $\nu_{\max }$ and $\ell_{\max }$ and convergence of the calculations is checked for increasing values of $\nu_{\max }$ and $\ell_{\max }$. Thus, the equations for the coefficients can be written in matrix form as

$$
\mathrm{i} \hbar \frac{d}{d t}|\Psi(t)\rangle=\left[\mathbf{H}_{0}-\boldsymbol{\mu} \varepsilon(t)\right]|\Psi(t)\rangle
$$

where the time-dependent state $|\Psi(t)\rangle$ is a column vector of dimension $\nu_{\max } \times\left(\ell_{\max }+1\right)$ composed of the expansion coefficients written in the following order: $a_{00}, a_{10} \ldots, a_{\nu_{\max } 0}, a_{01}, a_{11}, \ldots, a_{\nu_{\max } \ell_{\max }}$. The unperturbed Hamiltonian $\mathbf{H}_{0}$ is a diagonal matrix containing the corresponding rotational-vibrational energies $E_{\nu \ell}$, while the dipole matrix $\boldsymbol{\mu}$ has matrix elements given by

$$
(\boldsymbol{\mu})_{i j}=\left\langle\phi_{\nu^{\prime} \ell^{\prime}}|\mu| \phi_{\nu \ell}\right\rangle \times\left[p_{\ell} \delta_{\ell \ell^{\prime}+1}+p_{\ell-1} \delta_{\ell \ell^{\prime}-1}\right],
$$

with $i=\nu+\ell \nu_{\max }$ and $j=\nu^{\prime}+\ell^{\prime} \nu_{\max }$.

In order to solve Eq. (8), the corresponding propagator $U(t, 0)$ can be written in terms of a sequence of propagators $U(t+\Delta t, t)$ over a short time step $\Delta t$. The short-time propagator can in turn be approximated by the second order split-operator expression ${ }^{8}$

$$
U(t+\Delta t, t) \approx \mathrm{e}^{-\mathrm{i} \mathbf{H}_{0} \Delta t / 2 \hbar} \mathrm{e}^{\mathrm{i} \boldsymbol{\mu} \varepsilon(t) \Delta t / \hbar} \mathrm{e}^{-\mathrm{i} \mathbf{H}_{0} \Delta t / 2 \hbar}
$$

leading to the evolution of the state $|\Psi(t+\Delta t)\rangle=U(t+\Delta t, t)|\Psi(t)\rangle$.

Calculation of the photoassociation probability. Solving the radial equation (5) for a particular value of $\ell$ yields a certain number $N_{\ell}$ of bound states designated by $\left|\phi_{\nu \ell}^{\mathrm{B}}\right\rangle$ with energy $E_{\nu \ell}^{\mathrm{B}}$ and a certain number $\nu_{\max }-N_{\ell}$ of unbound levels designated by $\left|\phi_{\nu \ell}^{\mathrm{U}}\right\rangle$ with energy $E_{\nu \ell}^{\mathrm{U}}$. In particular, for $\ell=0$ the number of bound vibrational levels $N_{0}$ is given by the largest integer part of $\zeta$, which is determined by the relation $2 \zeta+1=\left(8 M D_{e}\right)^{1 / 2} /(\hbar \alpha)$. Thus, the wavefunction can be written in two parts, one involving the bound states and the other involving the unbound states,

$$
|\Psi(t)\rangle=\sum_{\ell=0}^{\ell_{\max }} \sum_{\nu=1}^{N_{\ell}} a_{\nu, \ell}(t)\left|\phi_{\nu \ell}^{\mathrm{B}}\right\rangle+\sum_{\ell=0}^{\ell_{\max }} \sum_{\nu=N_{\ell}+1}^{\nu_{\max }} a_{\nu, \ell}(t)\left|\phi_{\nu \ell}^{\mathrm{U}}\right\rangle .
$$

The gas at full thermal equilibrium will have some fraction of diatomic molecules present. However, we restrict to the remaining unbound atoms or, equivalently, to a nascent gas consisting solely of atoms in translational thermal equilibrium. Therefore, before the laser is turned on, the density operator can be written as ${ }^{9}$

$$
\rho(0)=\frac{1}{Z} \sum_{\ell=0}^{\ell_{\max }} \sum_{\nu=N_{\ell}+1}^{\nu_{\max }} a_{\nu, \ell}(t) \mathrm{e}^{-\beta E_{\nu \ell}^{\mathrm{U}}}\left|\phi_{\nu \ell}^{\mathrm{U}}\right\rangle\left\langle\phi_{\nu \ell}^{\mathrm{U}}\right|,
$$

where the summation is performed over all scattering levels.

The photoassociation probability $P_{\nu \ell}^{\mathrm{B}}\left(t_{f}\right)$ into a single rovibrational bound level $\left|\phi_{\nu \ell}^{\mathrm{B}}\right\rangle$ can be determined from

$$
P_{\nu \ell}^{\mathrm{B}}\left(t_{f}\right)=\operatorname{Tr}\left[\left|\phi_{\nu \ell}^{\mathrm{B}}\right\rangle\left\langle\phi_{\nu \ell}^{\mathrm{B}}\right| U\left(t_{f}, 0\right) \rho(0) U^{\dagger}\left(t_{f}, 0\right)\right],
$$

where $U(t, 0)$ is the time-evolution operator satisfying the Schödinger equation for the complete Hamiltonian $H=H_{0}+H_{1}$. Substituting the initial density operator in the above expression yields

$$
P_{\nu \ell}^{\mathrm{B}}\left(t_{f}\right)=\frac{1}{Z} \sum_{\ell=0}^{\ell_{\max }} \sum_{\nu=N_{\ell}+1}^{\nu_{\max }} a_{\nu, \ell}(t) \mathrm{e}^{-\beta E_{\nu \ell}^{\mathrm{U}}}\left|\left\langle\phi_{\nu \ell}^{\mathrm{B}}\left|U\left(t_{f}, 0\right)\right| \phi_{\nu \ell}^{\mathrm{U}}\right\rangle\right|^{2} .
$$

We define the total photoassociation probability $P^{\mathrm{B}}\left(t_{f}\right)$ summing $P_{\nu \ell}^{\mathrm{B}}\left(t_{f}\right)$ over all bound states,

$$
P^{\mathrm{B}}\left(t_{f}\right)=\sum_{\ell=0}^{\ell_{\max }} \sum_{\nu=1}^{N_{\ell}} P_{\nu \ell}^{\mathrm{B}}\left(t_{f}\right)
$$




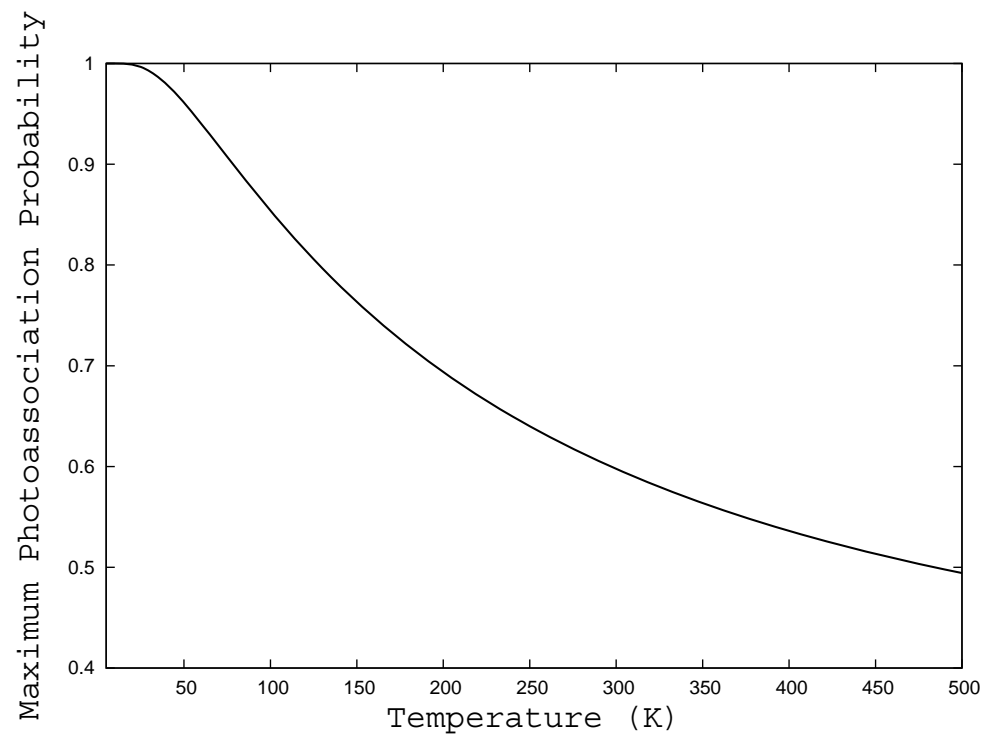

Figure 1. Upper bound for the total photoassociation probability $P_{\max }^{\mathrm{B}}$ as a function of the temperature. $\ell_{\max }=10$ and $\nu_{\max }=100$.

Upper bound for the total photoassociation probability. The very structure of quantum mechanics imposes constrains on the temporal variation of observable properties of a mixed state. ${ }^{10,11}$ These constrains, called kinematical constrains, limit the maximum attainable expectation value of observables. This is the case of the above defined photoassociation probability. Assuming the system can be treated effectively as having a finite number of levels and assuming its full controllability, the maximum possible photoassociation yield $P_{\max }^{\mathrm{B}}$ can be obtained as being

$$
P_{\max }^{\mathrm{B}}=\frac{1}{Z} \sum_{\ell=0}^{\ell_{\max }} \sum_{\nu=1}^{N_{\ell}} \mathrm{e}^{-\beta E_{\nu \ell}^{\mathrm{U}}},
$$

where the summation is performed over the number of bound levels, while the summands depend on the energy of the unbound levels. Comparing with Eqs. (14) and (15), the maximum photoassociation yield is achieved when the transitions $\left|\left\langle\phi_{\nu \ell}^{\mathrm{B}}\left|U\left(t_{f}, 0\right)\right| \phi_{\nu \ell}^{\mathrm{U}}\right\rangle\right|^{2}$ from the lowest unbound levels to the bound levels are one.

\section{NUMERICAL RESULTS}

Parameters corresponding to the $\mathrm{OH}$ molecule have been chosen. ${ }^{3,12}$ The Morse oscillator parameters are $D_{e}=0.1994$ a.u., $r_{e}=1.821$ a.u., $\alpha=1.189$ a.u. and $M=1728$ a.u., while the parameters of the permanent dipole function are $q=1.6 \mathrm{a} . \mathrm{u}$. and $r_{d}=1.134 \mathrm{a}$.u.. The infinite barrier that leads to the discretization of the unbound states is placed at $r=75 \mathrm{a}$.u. The eigenvalue equation (5) has been solved by the Fourier Grid Hamiltonian method. ${ }^{13}$

Figure 1 shows the photoassociation upper bound $P_{\max }^{\mathrm{B}}$ as a function of the temperature with the calculations parameters set to $\ell_{\max }=10$ and $\nu_{\max }=100$. It can be noted that the maximum possible yield decreases as the temperature increases. This feature is similar to the one previously observed in a $1 \mathrm{D}$ model $^{3}$ and reflects the fact that as $T \rightarrow \infty$, more unbound states are populated and the partition function $Z$ increases comparatively to $\mathrm{e}^{-\beta E_{\nu \ell}^{\mathrm{U}}}$ summed over all the bound states.

Figure 2 presents the total photoassociation probability $P_{\mathrm{B}}\left(t_{f}\right)$ for $t_{f}=0.5 \mathrm{ps}$ and the temperature set to $T=100 \mathrm{~K}$ and $T=300 \mathrm{~K}$. It is observed a number of peaks specially for frequencies below $\omega=2000 \mathrm{~cm}^{-1}$, indicating that high frequencies are not appropriate for inducing photoassociation, at least in this simplified model system. This can be explained based on the Boltzmann factors that remove the highly excited unbound states and also from the fact that the bound-unbound couplings decreases for increasing unbound energy. ${ }^{12}$ We additionally note that the higher obtained photoassociation probability for the two temperatures is considerably 


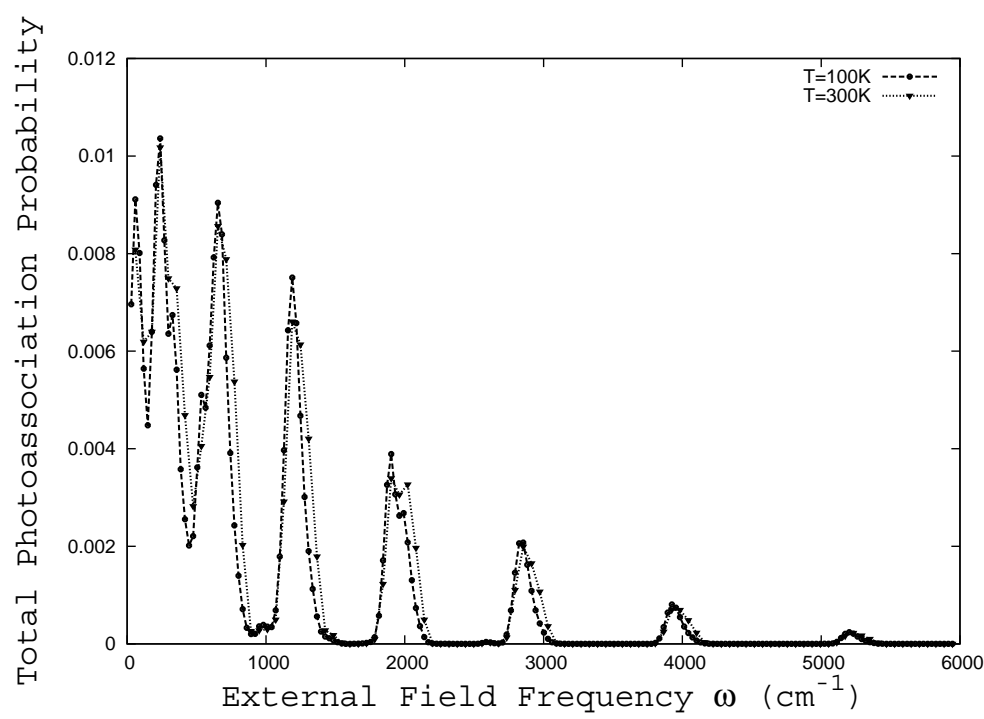

Figure 2. Total photoassociation probability $P^{\mathrm{B}}\left(t_{f}\right)$ as a function of the external field carrier frequency $\omega$ for $T=100 \mathrm{~K}$ and $T=300 \mathrm{~K}, \epsilon_{0}=80 \mathrm{MVcm}^{-1}$ and $t_{f}=0.5 \mathrm{ps} . \ell_{\max }=1$ and $\nu_{\max }=100$.

lower than the photoassociation upper bound by more than an order of magnitude. Figure 3 shows the total photoassociation probability as a function of the external field amplitude $\epsilon_{0}$ with fixed frequency $\omega=1189 \mathrm{~cm}^{-1}$ and for three temperatures. $P^{\mathrm{B}}\left(t_{f}\right)$ can be raise with higher amplitudes, however it is still much smaller than the upper bound. We note that the overall photoassociation probability is smaller for the higher temperature, which is compatible with the analysis of the maximum photoassociation yield.

\section{CONCLUSION}

We have investigated the photoassociation in a thermal gas of atoms including the rotational degree of freedom. Based on the assumption of full system controllability, the maximum possible photoassociation yield has been deduced. It has been found that the photoassociation upper bound decreases for increasing temperature in agreement with previous 1D calculations. Furthermore, the obtained photoassociation probability resulting from numerical calculations with fixed-shape pulses is considerably lower than the maximum photoassociation yield. This fact suggests that different kinds of pulse shapes might be more appropriate to induce photoassociation, such as up-chirped pulses and that quantum optimal control calculations must be performed in order to achieve the photoassociation upper bound.

\section{REFERENCES}

[1] J. Weiner, V. S. Bagnato, S. Zilio, and P. S. Julienne, "Experiments and theory in cold and ultracold collisions," Rev. Mod. Phys. 71, pp. 1-85, Jan 1999.

[2] J. Ulmanis, J. Deiglmayr, M. Repp, R. Wester, and M. Weidemller, "Ultracold molecules formed by photoassociation: Heteronuclear dimers, inelastic collisions, and interactions with ultrashort laser pulses," Chemical Reviews 112(9), pp. 4890-4927, 2012.

[3] E. F. de Lima, T.-S. Ho, and H. Rabitz, "Laser-pulse photoassociation in a thermal gas of atoms," Phys. Rev. A 78, p. 063417, Dec 2008.

[4] L. Rybak, Z. Amitay, S. Amaran, R. Kosloff, M. Tomza, R. Moszynski, and C. P. Koch, "Femtosecond coherent control of thermal photoassociation of magnesium atoms," Faraday Discuss. 153, pp. 383-394, 2011.

[5] U. Marvet and M. Dantus, "Femtosecond photoassociation spectroscopy: coherent bond formation," Chemical Physics Letters 245(45), pp. 393 - 399, 1995. 


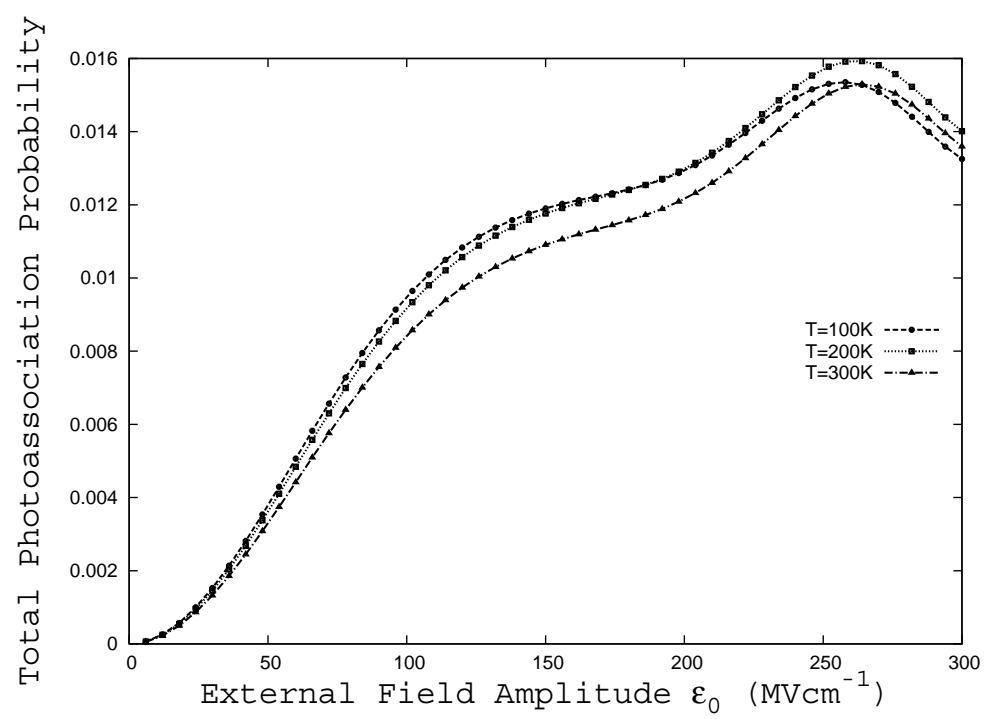

Figure 3. Total photoassociation probability $P^{\mathrm{B}}\left(t_{f}\right)$ as a function of the external field amplitude $\epsilon_{0}$ for $T=100 \mathrm{~K}, 200 \mathrm{~K}$ and $300 \mathrm{~K}, \omega_{0}=1189 \mathrm{~cm}^{-1}$ and $t_{f}=0.5 \mathrm{ps} . \ell_{\max }=1$ and $\nu_{\max }=100$.

[6] E. F. de Lima, T.-S. Ho, and H. Rabitz, "Solution of the schrödinger equation for the morse potential with an infinite barrier at long range," Journal of Physics A: Mathematical and Theoretical 41(33), p. 335303, 2008.

[7] S. Chelkowski and A. D. Bandrauk, "Control of molecular vibrational excitation and dissociation by chirped intense infrared laser pulses. rotational effects," The Journal of Chemical Physics 99(6), pp. 4279-4287, 1993.

[8] E. F. de Lima, T.-S. Ho, and H. Rabitz, "Optimal laser control of molecular photoassociation along with vibrational stabilization," Chemical Physics Letters 501(46), pp. 267 - 272, 2011.

[9] C. P. Koch, R. Kosloff, E. Luc-Koenig, F. Masnou-Seeuws, and A. Crubellier, "Photoassociation with chirped laser pulses: calculation of the absolute number of molecules per pulse," Journal of Physics B: Atomic, Molecular and Optical Physics 39(19), p. S1017, 2006.

[10] M. D. Girardeau, S. G. Schirmer, J. V. Leahy, and R. M. Koch, "Kinematical bounds on optimization of observables for quantum systems," Phys. Rev. A 58, pp. 2684-2689, 1998.

[11] M. D. Girardeau, M. Ina, S. Schirmer, and T. Gulsrud, "Kinematical bounds on evolution and optimization of mixed quantum states," Phys. Rev. A 55, pp. R1565-R1568, 1997.

[12] M. V. Korolkov, G. K. Paramonov, and B. Schmidt, "State-selective control for vibrational excitation and dissociation of diatomic molecules with shaped ultrashort infrared laser pulses," The Journal of Chemical Physics 105(5), pp. 1862-1879, 1996.

[13] C. C. Marston and G. G. Balint-Kurti, "The fourier grid hamiltonian method for bound state eigenvalues and eigenfunctions," The Journal of Chemical Physics 91(6), pp. 3571-3576, 1989. 